\title{
PHILelogite
}

Philologica Canariensia 25 (2019), pp. 66-85

DOI: 10.20420/PhilCan.2019.258

Recibido: 18 de febrero de 2019; aceptado: 24 de mayo de 2019

Publicado: 27 de julio de 2019

\section{DESAJUSTES ENTRE EL SIGNIFICADO Y LA FORMA EN PREGUNTAS ENCUBIERTAS. EL ANÁLISIS SINTÁCTICO DESDE EL SIGNIFICADO*}

\author{
EDITA GUTIÉRREZ RODRÍGUEZ \\ Universidad Complutense de Madrid
}

\author{
PILAR PÉREZ OCÓN \\ Universidad de Castilla-La Mancha
}

\begin{abstract}
RESUMEN: Las preguntas encubiertas son sintagmas nominales definidos que tienen un significado oracional, en lugar del empleo referencial típico de los sintagmas encabezados por el artículo definido. En este trabajo ofrecemos, en primer lugar, un repaso de las cuestiones más relevantes sobre las preguntas encubiertas: tipos, significado, comportamiento sintáctico y análisis. Nuestro objetivo principal, sin embargo, es investigar cómo se puede enseñar gramática de una manera distinta, que llegue a la forma desde el significado. Mostramos aquí por qué los sintagmas nominales de interpretación proposicional son un buen instrumento para ello. Explicamos en concreto cómo se pueden emplear estos sintagmas para reflexionar sobre conceptos como la composicionalidad, la selección, la diferencia entre gramaticalidad y aceptabilidad, o para comprender el significado de sintagmas y oraciones. Hacemos también una propuesta de actividades empleando métodos innovadores que favorezcan la reflexión y el estudio científico del lenguaje.
\end{abstract}

PALABRAS CLAVE: pregunta encubierta, artículo con valor enfático, enseñanza de la gramática, significado proposicional, desajuste

Mismatches between meaning and form in concealed questions.

The syntactic analysis from the perspective of meaning

\begin{abstract}
Concealed questions are definite noun phrases that have a propositional meaning, instead of the typical referential use of the phrases headed by the definite article. In this paper we offer first a review of the most important aspects about concealed questions: types, meaning, syntactic behavior, and analysis. Our main objective, however, is to investigate how grammar can be taught in a different way (from meaning to form). We show here why concealed questions are a good instrument for this purpose. We explain, in particular, how these phrases can be used to think about concepts such as compositionality, selection, the difference between grammaticality and acceptability, or to understand the different meaning of a nominal phrase and a sentence. We also propose activities using innovative methods that favor reflection and the scientific study of language. KEYWORDS: concealed questions, emphatic determiner, grammar teaching, propositional meaning, mismatch
\end{abstract}

1. El SIGNIFICADO DE LAS CATEGORÍAS GRAMATICALES: LLEGAR A LA GRAMÁTICA DESDE EL SIGNIFICADO

En los últimos cincuenta años del siglo $\mathrm{XX}$, algunas de las teorías lingüísticas más importantes, como el distribucionalismo americano o el primer generativismo, señalaron la 
supremacía de la forma sobre el significado y la necesidad de emplear pruebas formales en el análisis lingüístico. Como otros cambios en la ciencia lingüística, esta desconfianza hacia el significado se trasladó a la enseñanza secundaria y, como consecuencia de ello, una parte importante de la enseñanza de la gramática ha consistido en aprender a manejar pruebas formales que permitan, por ejemplo, diferenciar entre funciones sintácticas.

Creemos que, si el empleo de pruebas formales se acompaña de reflexiones sobre el significado, se puede evitar que el análisis gramatical se convierta en un etiquetado ciego de categorías y funciones. En los últimos años cada vez más lingüistas han subrayado la necesidad de incluir el significado en la enseñanza de la gramática, de manera que se vean las categorías y construcciones gramaticales como una forma de construir el significado en las lenguas (Escandell y Leonetti, 2011; Brucart, 2014; Bosque y Gallego, 2016; Leonetti 2017).

En este trabajo nos queremos centrar en el estudio de las preguntas encubiertas (ing. concealed questions), o sintagmas nominales de interpretación proposicional, del tipo de (1a). Se trata de sintagmas nominales que tienen la interpretación de una oración, en lugar de un significado típicamente nominal:

(1) a. No te imaginas la casa que tiene en la sierra

b. Visité la casa que tiene en la sierra

Así, en (1a) el sintagma nominal la casa que tiene en la sierra no tiene el mismo significado que en (1b). En (1b) se trata de un sintagma nominal definido que es referencial y, por tanto, señala a un objeto del mundo. En (1a), en cambio, el sintagma nominal se podría parafrasear por una oración exclamativa del tipo 'Qué casa tan \{bonita/grande\} tiene en la sierra'.

Los objetivos de este trabajo son tres. Por un lado, queremos ofrecer un estado de la cuestión claro y actualizado sobre las preguntas encubiertas. Por otro lado, queremos mostrar que este tipo de construcciones son un buen instrumento para establecer la relación entre la forma y el significado, y para reflexionar sobre qué tipo de significado tienen un sintagma nominal y una oración subordinada. Finalmente, nuestro tercer objetivo es investigar cómo se puede enseñar gramática de una forma distinta y hacer una propuesta de actividades sobre los sintagmas nominales de interpretación proposicional desde la innovación metodológica.

En concreto, defenderemos que este tipo de sintagmas pueden servir para que los estudiantes sean conscientes de los siguientes principios y cuestiones gramaticales:

a. La selección semántica (ing. semantic selection), es decir, la idea de que el verbo impone requisitos semánticos sobre su objeto (Grimshaw, 1979).

b. La selección categorial (ing. categorial selection), también llamada selección sintáctica o subcategorización, es decir, la idea de que el verbo también impone requisitos categoriales sobre su objeto.

c. El tipo de significado de los sustantivos y de las oraciones. Hay que transmitir a los alumnos que cada clase de sintagma tiene un tipo específico de significado. Los sintagmas nominales denotan entidades como objetos, personas, lugares o momentos, mientras que las oraciones sustantivas denotan entidades más abstractas, como hechos, acciones, situaciones o estados de cosas (NGLE, 2009, § 43.1e). En el acercamiento distribucional, muy presente en los libros de texto de secundaria, se señala que los sintagmas nominales y las oraciones subordinadas sustantivas tienen la misma distribución. Esta afirmación es solo parcialmente cierta, pues hay algunos 
predicados que aceptan sintagmas nominales, pero no oraciones sustantivas, de complemento (Comió \{un bocadillo/*que quería\}). Pocas veces se hace la reflexión sobre cómo el diferente significado de sustantivos $\mathrm{y}$ oraciones condiciona su distribución.

d. Por último, la idea de que la gramática es un conjunto de reglas, que hay cuestiones que se deben y se pueden explicar y comportamientos que se pueden predecir. En el caso de las preguntas encubiertas, el significado de estas no es el que se espera de un sintagma nominal. Este es el primer paso de la investigación científica, una observación atenta de la realidad para darse cuenta de que hay algo que requiere una explicación. Los estudiantes tienen que saber que los gramáticos no hacemos listas de excepciones, sino que los comportamientos que aparentemente lo son tienen una explicación que quizá todavía no conocemos y que hay que encontrar.

Este trabajo se organiza de la siguiente manera. En el $\S 2$ haremos un repaso sobre los sintagmas nominales de interpretación proposicional: qué son, qué significado tienen, qué tipos hay y cómo se pueden analizar. En el $\S 3$ explicaremos por qué creemos que se debería trabajar con estas construcciones en secundaria. En el $\S 4$ ofreceremos un pequeño muestrario de ejercicios de distinto tipo para trabajar con estas construcciones en las aulas. Por último, en el $\S 5$ reuniremos nuestras conclusiones.

\section{LAS PREGUNTAS ENCUBIERTAS. DEFINICIÓN Y PROPIEDADES BÁSICAS}

En este apartado haremos un repaso de la bibliografía que nos sirva de marco teórico para nuestra propuesta didáctica. Empezaremos ofreciendo una definición y una descripción de los tipos básicos de preguntas encubiertas en el § 2.1. En el § 2.2 veremos el tipo de significado que tienen los sintagmas nominales de interpretación proposicional. En el § 2.3 precisaremos qué predicados toman sintagmas nominales de interpretación proposicional y qué tipo de nombres pueden aparecer en la construcción. El § 2.4 se dedica a repasar el comportamiento sintáctico específico de estas construcciones. Terminaremos en el $\S 2.5$ con los análisis de enfoque sintáctico y pragmático que se han ofrecido para estas construcciones.

\subsection{Definición y tipos de preguntas encubiertas}

Una pregunta encubierta -o sintagma nominal de interpretación proposicional- es un sintagma nominal (SN) que tiene una interpretación semejante a la de una oración interrogativa (2) o exclamativa indirecta (3): ${ }^{1}$

(2) a. Juan no sabía la hora

b. No recuerdo el vino que le gusta más

(3) a. Me sorprendió el ganador del premio

b. Son increíbles los libros que lee

Las oraciones de (2)-(3) tienen un significado equivalente a las de (4)-(5), donde una oración interrogativa o exclamativa indirecta sustituye al SN: 
(4) a. Juan no sabía qué hora era

b. No recuerdo qué vino le gusta más

(5) a. Me sorprendió quién era el ganador del premio

b. Es increíble qué libros lee

Según la estructura interna de los sintagmas, vamos a diferenciar entre SN de interpretación proposicional simples ( $S N$ simples), que contienen un sustantivo y opcionalmente modificadores no oracionales (6), y SN de interpretación proposicional complejos ( $S N$ complejos), que necesitan una oración para que se dé la interpretación proposicional (7):

(6) a. Le preguntó la hora

b. Juan sabe el camino

c. La solución es evidente

d. Les sorprendió el tamaño de la cama

(7) a. No está segura del libro que compró

b. Es increíble las ganas que tiene de ir

c. Preguntó la gente que venía

d. Le sorprendió la mesa que eligió

Los $S N$ simples se forman con el artículo definido (8a) y también con el posesivo (8b), pero no con el demostrativo (8c), mientras que los $S N$ complejos se construyen únicamente con el artículo definido y una oración encabezada por que (8d):

(8) a. No sé la dirección 'No sé cuál es la dirección'

b. No sé su dirección 'No sé cuál es su dirección'

c. * No sé esta dirección

d. * Le sorprendió esta mesa que eligió

Los SN complejos (9) pierden, en la mayoría de los casos, la lectura proposicional en ausencia de la oración encabezada por que y, por ello, las oraciones que los contienen se convierten en agramaticales si el predicado que selecciona el sintagma nominal exige semánticamente un complemento de naturaleza oracional:

(9) a. ??No está segura del libro

b. *Es increíble las ganas

c. *Preguntó la gente

d. *Le sorprendió la mesa

\subsection{El significado de las preguntas encubiertas}

Ya hemos señalado que las preguntas encubiertas son SN definidos que no tienen las propiedades referenciales (anafóricas o deícticas) típicas de los SN definidos. Vamos a explicar qué tipo de significado tienen estos sintagmas y para ello diferenciaremos entre: ${ }^{2}$ 
a. SN complemento de un predicado interrogativo: Dime la hora 'Dime qué hora es'; Dime la hora que es 'Dime qué hora es'.

b. SN complemento de un predicado exclamativo: Me sorprendió la capital de Turquía 'Me sorprendió cuál era la capital de Turquía'; Me sorprendió los amigos que tenía 'Me sorprendió cuántos amigos tenía'.

No hay, en cambio, sintagmas nominales con una interpretación correspondiente al de una oración asertiva, como en Dijo su llegada 'Dijo que vendría' o en Creo su tardanza 'Creo que llegará tarde' (ejemplos de Bosque, 1989, 97).

El tipo de significado de los complementos de los predicados interrogativos y exclamativos es distinto (NGLE, 2009, 43.7s). Así, la oración interrogativa indirecta No sé cómo lo hizo contrasta con la exclamativa Es terrible cómo lo hizo. En el primer caso, la subordinada introduce una situación con una variable de modo cuyo valor está por determinar. En el segundo caso, en cambio, el hablante ya tiene acceso a la información sobre cómo lo hizo ${ }^{3}$ y se evalúa esta información, de manera que se le asigna un valor extremo. Los mismos significados tienen los SN de interpretación proposicional. Así, en No sé la solución se introduce una variable que se corresponde con cuál es la solución, mientras que en No sabes la solución que encontraron, con lectura exclamativa, el hablante ya conoce la solución y la evalúa.

Vamos a estudiar con más detalle el significado de los SN interrogativos y exclamativos, distinguiendo entre SN simples y complejos. Los SN simples tienen solo la lectura llamada existencial, que equivale a una oración copulativa de identidad, como se refleja en las paráfrasis de (10), con predicado interrogativo, y de (11), con predicado exclamativo:4

(10) a. No sabe la capital de Turquía

'No sé cuál es la capital de Turquía'

b. No sabe los números primos

'No sabe cuáles son los números primos'

(11) a. Me sorprendió la capital de Turquía

'Me sorprendió cuál era la capital de Turquía'5

b. Me sorprendieron las soluciones de Juan al problema

'Me sorprendió cuáles fueron las soluciones de Juan al problema'

Como se ve en los ejemplos de (11), los SN simples exclamativos tienen únicamente la lectura existencial, y no parecen tener las lecturas ponderativas que toman los SN complejos y que describiremos a continuación.

En los SN complejos, el artículo definido está usado de manera cuantificativa o enfática (Torrego, 1988; Brucart, 1999, § 7.4.2; Leonetti, 1999, § 12.1.2.7; NGLE, 2009, §§ 14.9d, 22.12l, 42.16; Bosque, 2017b, § 5.1.2). Este artículo aparece tanto en construcciones interrogativas (12a) como en exclamativas indirectas (12b), y también en oraciones exclamativas independientes (12c), pero no en interrogativas directas (12d):

(12) a. No sé los libros que ha traído

b. Me sorprende los libros que ha traído

c. iLos libros que ha traído!

d. \#¿̇Los libros que lee? 
En el caso de las exclamativas independientes (12c), la interpretación del sintagma nominal es ponderativa o enfática, y puede tener un valor cuantitativo ('iCuántos libros ha traído!') o cualitativo ('iQué libros ha traído!'). La interpretación exclamativa o ponderativa expresa un valor extremo, que puede estar orientado en sentido positivo o negativo ('iQué libros tan \{buenos/horribles\}!'), como se señala en Leonetti (1999, § 12.1.2.7), NGLE (2009, § 42.16h) o Bosque (2017b, capítulo 1), entre otros.

En cuanto a las preguntas encubiertas complejas (13), las que son complemento de predicados exclamativos tienen, como es esperable, una lectura cualitativa, que puede ser positiva o negativa, y también una lectura cuantitativa: ${ }^{6}$

(13) Me sorprende los libros que ha leído

'Me sorprende qué libros (tan \{buenos/malos\}) ha leído'

'Me sorprende cuántos libros ha leído'7

Las preguntas encubiertas complejas complemento de predicados interrogativos (14), en cambio, tienen solo la lectura existencial y no parecen tener ni la lectura cuantitativa ${ }^{8}$ ni la cualitativa, que se asocian con la ponderación propia de las oraciones exclamativas:

(14) a. No sé los libros que lee

'No sé qué libros lee' / 'No sé cuáles son los libros que lee'

\#'No sé cuántos libros lee'

\#'No sé qué libros tan \{buenos/malos\} lee’

b. Me pregunto los sitios que ha visitado

'Me pregunto qué sitios ha visitado'

\#'Me pregunto cuántos sitios ha visitado’

En los SN complemento de predicados interrogativos se habla también de artículo enfático, aunque, como hemos señalado, el sintagma no tiene propiamente un significado enfático en el sentido de que no expresa un valor extremo. Sería más apropiado aquí hablar de artículo cuantificativo o artículo como operador, pues lo que sí tiene en común el artículo en SN complejos complemento de predicados interrogativos y exclamativos es su empleo como una palabra $q u$ - (véase el § 2.5).

2.3. Predicados y sustantivos que dan lugar a la lectura proposicional de los sintagmas nominales

Los sintagmas nominales toman la interpretación proposicional cuando son complemento de ciertos predicados, como descubrir, imaginar, olvidar, recordar, revelar, saber, etc., que parecen contribuir de alguna manera al significado interrogativo o exclamativo del SN. En general, son predicados que seleccionan oraciones interrogativas o exclamativas indirectas de complemento, aunque, como se señala en Bosque (1982, 32), no todos los predicados que seleccionan este tipo de complemento aceptan SN de interpretación proposicional. Así, ciertos verbos como saber, decir o depender parecen aceptarlos más fácilmente que otros como descubrir, olvidar u observar.

En la NGLE (2009, 43.7j) se ofrece una clasificación de los predicados que seleccionan interrogativas indirectas y se señala que se agrupan en unas pocas clases que tienen en común 
el concepto de información, entendiendo este concepto en sentido amplio para abarcar contenidos pensados, conocidos, supuestos, etc. Así, estos predicados pueden expresar solicitud de información (preguntar), posesión de información (saber), ausencia o pérdida de información (ignorar, olvidar), etc. En cuanto a los predicados que introducen exclamativas indirectas, su número es mucho más reducido. En Gutiérrez-Rexach y Andueza $(2017,181)$ se señala que los predicados que toman exclamativas tienen en común que son predicados de emoción (ing. emotive predicates), pues las oraciones exclamativas se usan básicamente para expresar actitudes del hablante con un contenido emotivo (ser importante, ser una locura, dar rabia, etc.).

En lo que respecta al tipo de sustantivo que aparece como núcleo del $\mathrm{SN}$, conviene aquí diferenciar entre los SN simples y los SN complejos (véase § 2.1). En el caso de los SN simples, no todos los nombres dan lugar a la lectura proposicional:

(15) a. Le preguntó la hora

b. *Le preguntó la sopera

(16) a. Juan sabe el camino

b. *Juan sabe la mesa

(17) a. Decidieron el tamaño de la cama

b. *Decidieron la cama 9

En la NGLE (2009, § 43.8d) se señala que muchos de los sustantivos que toman lectura proposicional son relacionales y pueden aparecer en oraciones copulativas con el interrogativo cuál en usos no anafóricos ni deícticos de este interrogativo. Así, en (18a) cuál tiene necesariamente una lectura anafórica o deíctica, mientras que en (18b) no es necesariamente así; sobre el uso no anafórico del interrogativo cuál, véase NGLE (2009, § 22.14e). El sustantivo tamaño, frente a cama, toma lecturas oracionales:

(18) a. ¿Cuál es la cama de Luis?

b. ¿Cuál es el tamaño de la cama de Luis?

Según Bosque (2017a, 29), los nombres que aparecen en estas construcciones son sustantivos que dan lugar a "expresiones identificadas por su nombre", o bien que se identifican con valores cuantitativos (precio, edad, fecha, etc.). Así, en No recuerdo la capital de Eslovenia, en la lectura proposicional se alude a la identificación del nombre de la capital de Eslovenia, mientras que en No recuerdo la edad de Juan, se busca identificar el valor cuantitativo correspondiente, es decir, los años que tiene Juan. Más allá de lo dicho aquí, no resulta sencillo identificar el criterio que permite restringir la clase de sustantivos que dan lugar a la lectura de pregunta encubierta. ${ }^{10}$

En cuanto a los SN complejos, prácticamente cualquier sustantivo puede aparecer en esta construcción cuando incluye una oración encabezada por que (Vatrican, 2006, 268):

(19) a. * Me pregunto \{la cama/los libros/los amigos\}

b. Me pregunto la cama que compró

c. Me pregunto los libros que lee

d. Me pregunto los amigos que tiene 
Por tanto, en el caso de los SN complejos parece que es la construcción, y no el sustantivo, el responsable de la lectura oracional. Este comportamiento se puede explicar si los dos tipos de sintagmas se analizan de manera diferente (véase el § 2.5).

2.4. El comportamiento sintáctico de los sintagmas nominales de interpretación proposicional

Los sintagmas nominales de interpretación proposicional tienen un comportamiento sintáctico y semántico distinto de los sintagmas nominales que denotan una entidad. En primer lugar, si el predicado puede seleccionar complementos nominales y oraciones, el SN puede tener dos interpretaciones, la nominal y la oracional:

(20) Los alumnos estudiaron la solución al problema

En una interpretación, el SN es una pregunta encubierta y se entiende que los alumnos no conocen la solución ('Estudiaron cuál podía ser la solución al problema'). En la otra interpretación, el SN denota una entidad existente, por tanto, los alumnos conocen la solución y la estudian, por ejemplo, porque saben que va a caer en el examen.

En segundo lugar, el SN puede ser retomado por el clítico neutro (lo), en lugar del pronombre masculino o femenino (lo/la), en la interpretación proposicional (Bosque, 1982, 33; NGLE, 2009, § 43.8f):

(21) a. Me dijo la calle, pero lo olvidé (Bosque, 1982, 33)

b. ?Me dijo la calle, pero la olvidé

(22) a. No sabes bien los libros que le gustan

b. No lo sabes bien

c. *No los sabes bien

En cambio, si el SN tiene la interpretación de entidad, esta sustitución por el clítico neutro no es posible:

(23) a. *Arreglaron la calle, pero no lo asfaltaron

b. Arreglaron la calle, pero no la asfaltaron

(24) a. Vi los libros que le gustan

b. Los vi

c. \# Lo vi

En tercer lugar, cuando el SN que es complemento directo contiene un nombre de persona, la preposición $a$ es necesaria en la interpretación de entidad del SN, pero no lo es en la lectura proposicional (Bosque, 1989, 95):

(25) a. No recuerdo el autor de la novela

b. No recuerdo al autor de la novela

(26) a. Estudian el ministro que enviarán a la cumbre del cambio climático

b. Estudian al ministro que enviarán a la cumbre del cambio climático 
En (25a) y (26a) la interpretación del SN es proposicional y las oraciones se pueden parafrasear como 'No recuerdo quién es el autor de la novela' y 'Estudian qué ministro enviarán a la cumbre'. En cambio, en (25b) y (26b) la preposición fuerza la lectura de entidad, se sabe quién es el autor de la novela y a quién se va a enviar a la cumbre. En el primer caso, aunque se sabe quién es, no se le recuerda. En el segundo, se ha decidido quién va a ir, pero le están investigando, por ejemplo, para ver si es la persona adecuada.

En cuarto lugar, un SN plural sujeto de un predicado exclamativo puede concordar con el verbo en singular (como las oraciones) o en plural (como los sintagmas nominales) (Bosque, 1982, 26):

(27) a. Es increíble las películas que ha visto tu amigo

b. Son increíbles las películas que ha visto tu amigo

Si hay discordancia, ${ }^{11}$ la interpretación proposicional es la única posible, de manera que el SN de (27a) solo tiene la lectura 'Es increíble cuántas películas ha visto tu amigo' o 'Es increíble qué películas tan buenas (o malas) ha visto tu amigo'; en cambio, en (27b) solo es posible la lectura de entidad: hay unas películas de las que se está hablando y todas son increíbles. Se trata en tal caso de una oración declarativa, no exclamativa (Bosque, 1982, 26).

Además, también se puede producir discordancia de género si el predicado es un adjetivo, y el SN de interpretación proposicional, de nuevo, puede concordar en neutro (o masculino) (28a) en lugar de en femenino (28b):

(28) a. Es curioso las personas que has visto crecer bajo tu tutela

b. Son curiosas las personas que has visto crecer bajo tu tutela

En todos los casos, la discordancia se produce más raramente o no se produce si el SN aparece antepuesto al predicado: ${ }^{12}$

(29) a. ??Las películas que ha visto tu amigo es increíble

b. *Las personas que has visto crecer bajo tu tutela es curioso

En resumen, existe una relación entre la interpretación del SN, la posición que ocupa el sujeto y la concordancia verbal. Si el SN plural aparece pospuesto al verbo y concuerda en singular, tiene interpretación proposicional (30a). Cuando una oración es sujeto con este tipo de predicados, ocurre lo mismo: concuerda en singular y tiende a ocupar la posición posverbal como no marcada (Es sorprendente que siempre llegue tarde / Que siempre llegue tarde es sorprendente). Si el SN plural concuerda con el verbo en plural, se obtiene la interpretación de entidad (30b) y se prefiere la anteposición con un SN definido, aunque la posposición es posible también (Son sorprendentes los peligros que ha corrido este hombre) con la misma interpretación no oracional.

(30) a. Es sorprendente los peligros que ha corrido este hombre

b. Los peligros que ha corrido este hombre son sorprendentes

En cuanto a los predicados interrogativos, en Bosque $(1982,26)$ se señala que dan lugar a este tipo de discordancia con más dificultad: 
Aunque es cierto que existe un contraste entre las lecturas exclamativas y las interrogativas, también con predicados interrogativos se puede producir la discordancia, como en el ejemplo de (32). Claramente, hay muchos menos casos con discordancia en interrogativas, quizá porque hay menos predicados interrogativos del tipo $<$ ser + adjetivo $>$.

(32) Es misterioso la forma en que se desarrollan las cosas cuando te pasa algo así13

Por último, la posición de la preposición $a$ también puede ayudar a diferenciar entre la lectura nominal y la oracional (NGLE, 2009, § 42.16i-j). En (33a) y (34a) la lectura nominal no es posible y el hecho de que los predicados ser increíble y saber no exijan la preposición $a$ indica que el complemento de estos predicados es en estos ejemplos una oración (NGLE, 2009, $\S$ 22.12l), como veremos en el apartado 2.5. En cambio, en los ejemplos de (33b) y (34b) estamos ante sintagmas nominales (preguntas encubiertas con SN simples). Finalmente, los ejemplos de (33c) y (34c) son oraciones exclamativas e interrogativas estándar.

(33) a. Es increíble a la destreza que llegan algunos

b. Es increíble la destreza a que llegan algunos

c. Es increíble a qué destreza llegan algunos

(34) a. Nunca sé a la hora que salgo

b. Nunca sé la hora a la que salgo

c. Nunca sé a qué hora salgo

En resumen, hemos visto que los sintagmas nominales de interpretación proposicional, al menos los SN complejos, tienen muchas propiedades sintácticas que los diferencian de un sintagma nominal y que los asimilan a construcciones oracionales.

\subsection{Análisis sintácticos y pragmáticos de las preguntas encubiertas}

No queremos acabar este apartado dedicado a la descripción de las preguntas encubiertas sin hacer un resumen de las dos líneas principales de análisis que se han ofrecido para explicarlas. En la primera se considera que el complemento es una oración cuyo significado proposicional se sigue de su naturaleza sintáctica (véanse, por ejemplo, Plann, 1982; Bosque, 1984; Bosque y Moreno, 1990, nota 1; NGLE, § 43.8). En la segunda línea de análisis la pregunta encubierta no es una oración interrogativa, sino un SN y el significado proposicional se obtiene mediante una operación semántico-pragmática (véanse Harris et al., 2008; Nathan, 2006; Romero, 2006; Frana, 2017). El primer análisis puede explicar los SN de interpretación proposicional complejos (Me preguntó la hora que era) y el segundo, los simples (Me preguntó la hora).

I. EL ARTÍCULO COMO OPERADOR. En este análisis el artículo enfático es un operador ${ }^{14}$ semejante a una palabra $q u$-, de manera que la pregunta encubierta es en realidad un constituyente oracional y no nominal (NGLE, § 42.16). La forma que se considera una conjunción (y no un 
relativo) y el SN encabezado por el artículo enfático se desplaza al inicio de la oración desde su posición original:

(35) Le sorprende [o [las cosas] que dice las cosas]

Este análisis explica la interpretación de estos sintagmas nominales, por ejemplo, el hecho de que, a pesar de ir encabezados por el artículo definido, no se refieran a una entidad particular. También da cuenta de que estos SN se retomen con el clítico neutro y que no haya concordancia en caso de que el SN sea el sujeto, pues si el sujeto es oracional la concordancia se hace siempre en singular:

(36) Me sorprende los libros que lee

(37) a. Me sorprendió qué libros le gustan

b. Es increíble cuántos amigos tiene

Se explica, además, que un predicado como saber, que solo toma complementos oracionales, admita este tipo de sintagmas de complemento, y también el comportamiento con la preposición a en ejemplos como Es increíble a la hora que llegan sus hijos. Sin embargo, este análisis no puede explicar las preguntas encubiertas simples, fundamentalmente porque no hay una oración con la forma que en el interior del sintagma. Por ello, para estas parece más adecuado un análisis de tipo semántico-pragmático como el que vamos a presentar a continuación.

II. ANÁLISIS SEMÁNTICO-PRAGMÁTICO. Para derivar la reinterpretación que se produce con los SN simples de interpretación proposicional se han propuesto diversos procedimientos que cambian el tipo semántico del SN. Emplearemos el término coacción (ing. coercion) en sentido amplio para referirnos a la operación que permite que interpretemos un SN como una oración. ${ }^{15}$ Esta operación repara el desajuste semántico cambiando el significado de un elemento para que sea compatible con el significado del elemento que lo selecciona. En un ejemplo como El profesor no sabía el autor, el predicado saber, que exige complementos oracionales, "coacciona" al SN el autor, que pasa de denotar una entidad a interpretarse como una pregunta. Es importante señalar que este comportamiento no implica que estemos tratando con una oración interrogativa desde el punto de vista sintáctico, ya que la coacción del complemento no es un procedimiento sintáctico. ${ }^{16}$

Una prueba de que las preguntas ocultas se comportan como expresiones "coaccionadas" es que dan lugar a un efecto interpretativo sistemático y, por tanto, predecible, como veíamos en el § 2.3. Básicamente, la reparación del desajuste entre el verbo y su complemento tiene como resultado una interpretación proposicional de pregunta existencial: No sabe la hora 'No sabe qué hora es'.

Otro efecto interpretativo derivado de la reparación del desajuste tiene que ver con el carácter marcado de las preguntas encubiertas. A diferencia de lo que sucede con las expresiones agramaticales, las construcciones con desajustes semánticos son toleradas por el sistema gramatical, pero reciben una interpretación marcada que requiere un mayor esfuerzo de procesamiento. ${ }^{17}$ En Harris et al. (2008, 45-46) se demuestra con experimentos magnetoencefalográficos (MEG) y de rastreo ocular (eye-tracking) que las preguntas 
encubiertas son expresiones más costosas para los hablantes, precisamente por la necesidad de reparar el desajuste.

En resumen, hemos visto los dos análisis que se ofrecen para explicar las preguntas encubiertas. El análisis del artículo como operador y de la forma que como una conjunción puede dar cuenta de manera satisfactoria de las propiedades oracionales de los SN complejos y, en cambio, un análisis con coacción puede explicar las preguntas encubiertas simples.

\section{3. ¿POR QUÉ TRABAJAR CON LAS PREGUNTAS ENCUBIERTAS EN LA EDUCACIÓN SECUNDARIA?}

Tras examinar en el $\S 2$ las propiedades gramaticales de los SN de interpretación proposicional, vamos a investigar qué puede aportar la enseñanza de estas construcciones en la educación secundaria. Como vimos en la introducción, estas construcciones nos permiten introducir en las aulas algunos principios gramaticales básicos:

a) el principio de composicionalidad y, por tanto, la relación entre forma y significado

b) el significado de las categorías gramaticales, concretamente de los $\mathrm{SN}$ y las oraciones

c) los principios de selección (categorial y semántica) entre predicado y argumento

d) la diferencia entre corrección y gramaticalidad

\subsection{El principio de composicionalidad}

Las preguntas encubiertas pueden usarse para transmitir la idea de que debe haber una relación directa entre el significado de una frase y su forma y que, si esta relación no se establece, debe ser explicada de alguna manera. Las preguntas encubiertas de ejemplos como Preguntó la hora son un caso de "construcción no composicional". ${ }^{18} \mathrm{El}$ verbo preguntar selecciona oraciones interrogativas y no se espera que un SN aparezca como su complemento, ya que los SN denotan entidades (objetos, personas, lugares, etc.), mientras que las oraciones sustantivas denotan hechos, situaciones o estados de cosas.

Es necesario, por tanto, que los alumnos entiendan que necesitamos articular algún mecanismo para explicar cómo los hablantes "montamos" el significado de la secuencia Preguntó la hora y cómo un SN termina significando lo mismo que una oración (Preguntó qué hora era). No pretendemos que los estudiantes avancen explicaciones sobre cuestiones tan complejas; bastaría con que comprendieran que el etiquetado en categorías y funciones de la expresión Preguntó la hora no es suficiente para ver las propiedades interesantes que esconde esta oración.

\subsection{Selección semántica y selección categorial}

Detenernos en observar cómo se combinan los SN de interpretación proposicional con sus predicados resulta útil para mostrar que los predicados limitan el contenido semántico y la categoría sintáctica de sus argumentos. Estas restricciones de selección, como hemos señalado en el $\S 1$, son conocidas como selección s(emántica) y selección c(ategorial) y conforman la estructura argumental de un predicado. ${ }^{19}$ La agramaticalidad de los siguientes ejemplos se explica precisamente porque existe una infracción de los requisitos de selección. Un predicado como comprar selecciona $\mathrm{SN}$ y no oraciones, mientras que saber selecciona oraciones y no SN. ${ }^{20}$ 
(38) a. Compró un libro

b. *Compró que María leía

(39) a. Sabía que María llegaría tarde

b. *Sabía su retraso

Al comparar las oraciones *Sabía su retraso (39b) y Sabía la hora, constatamos que esta última es gramatical aunque no debería serlo, pues estamos combinando un predicado que exige oraciones (saber) con un SN. ${ }^{21} \mathrm{Si}$ los estudiantes comprenden que una oración tan simple como Sabía la hora es una anomalía, estarán empezando a entender que la gramática está basada en las relaciones que se establecen entre un elemento que selecciona y otro que es seleccionado. La pregunta que surge a raíz de la anterior observación es por qué la oración Sabía la hora es posible. La respuesta, como hemos visto en el § 2, no es sencilla, pero, con lo explicado en aquel apartado, un profesor podría esbozar una respuesta satisfactoria para los alumnos.

\subsection{Diferencia entre gramaticalidad y corrección}

Demasiadas veces se transmite a los estudiantes la idea de que la lengua es un conjunto de pautas que debemos seguir, como si fuera el código de la circulación. Si bien es cierto que los alumnos deben conocer la norma gramatical, no es menos cierto que deben saber cuándo una expresión anómala es un "fallo del sistema" (agramaticalidad) o es fruto de un rechazo sociolingüístico (incorrección).

En (40) podemos observar fenómenos de discordancia con preguntas encubiertas: en (40a) el adjetivo increíble es singular y el SN sujeto es plural, y en (40b) el nombre hora es femenino y el pronombre lo es neutro.

(40) a. Es increíble los libros que lee

b. Me dijo la horora, pero lo olvidé

Desde un enfoque prescriptivista, se diría que los ejemplos de (40) son incorrectos porque no hay concordancia, mientras que desde un acercamiento más descriptivo se explicaría la discordancia, ya que sería un reflejo de que los hablantes están interpretando el SN como una oración encubierta.

En resumen, estos datos permiten a los estudiantes reflexionar sobre el hecho de que existe un patrón regular detrás de una aparente irregularidad. El acercamiento que proponemos implica adoptar una actitud inquisitiva ante los fenómenos lingüísticos -¿̇por qué ocurre esto? - semejante a la que se fomenta en las aulas en las clases de ciencias. Este acercamiento no es incompatible con la conciencia de la norma y con la mejora de la competencia lingüística, pues trabajar con estas construcciones permite adquirir a los estudiantes una conciencia metalingüística que resulta de gran ayuda en el control de las discordancias.

\section{4. ¿CÓMO TRABAJAR CON LAS PREGUNTAS ENCUBIERTAS EN LA EDUCACIÓN SECUNDARIA?}

En el propio currículo de lengua castellana, la enseñanza de la gramática en secundaria se ve constantemente cuestionada, especialmente si no contribuye a adquirir las destrezas 
relacionadas con el uso correcto del lenguaje y con la comunicación..$^{22}$ El enfoque representado por Gramática Orientada a las Competencias $(\mathrm{GrOC})^{23}$ pretende superar esta tendencia antigramaticalista y propone enseñar gramática dando peso a otras competencias tradicionalmente asociadas al ámbito de las ciencias. A partir de esta línea de trabajo, vamos a plantear algunos ejercicios con las preguntas encubiertas.

\subsection{Actitudes y metodología}

En la línea de trabajos recientes (Escandell y Leonetti, 2011; Brucart, 2014; Leonetti, 2017), nuestra propuesta es estudiar los datos lingüísticos desde la intuición que los hablantes tienen sobre la lengua, empezando desde el significado para llegar a la forma y contextualizando los ejemplos si es necesario. Por otro lado, creemos que la enseñanza de la gramática debe acompañarse de una metodología basada en ejercicios que promuevan la reflexión. Se ha dicho ya suficientes veces que debemos huir de las actividades que consisten en etiquetar categorías y funciones de forma irreflexiva. En su lugar, deberíamos crear actividades para que los alumnos adoptaran una actitud más activa que les sirviera para detectar, a través de la observación y la experimentación, qué propiedades del sistema nos revelan determinados ejemplos. Deberíamos también ser capaces de crear ejercicios que permitieran a los alumnos inventar sencillas pruebas a favor o en contra de una explicación. Por último, nuestros ejercicios deberían ayudarles a formular generalizaciones que describan o expliquen el comportamiento de un conjunto de datos.

Si queremos que nuestros estudiantes descubran y conozcan el sistema lingüístico tendremos que cambiar las actitudes y la metodología con la que se ejercitan. Para ello es necesario, por un lado, que se entienda que "enseñar gramática" no es "enseñar a hablar" y, por otro, que se diseñen ejercicios que faciliten el descubrimiento de los principios del sistema gramatical. Esta metodología es presentada en Bosque y Gallego (2016) e incluye los siguientes tipos de ejercicios: a) análisis directo de secuencias breves; b) análisis inverso; c) análisis de secuencias agramaticales y de secuencias ambiguas; d) análisis de pares mínimos y de dobles pares mínimos; e) análisis de secuencias ambiguas; f) ejercicios de elección de análisis. En el § 4.2 vamos a diseñar una propuesta sobre las preguntas encubiertas basándonos en los ejercicios de análisis directo a partir de secuencias breves, análisis de secuencias agramaticales, análisis de pares mínimos y análisis de secuencias ambiguas.

\subsection{Propuesta de trabajo con las preguntas encubiertas}

Conviene mostrar, en primer lugar, que en una oración tan simple como (41) hay algo que comprender. Para diseñar el ejercicio es necesario partir de la intuición lingüística de los estudiantes, del significado de la oración, y no del análisis de sus componentes.

Me sorprendió el ganador de las elecciones [Bosque, 1989, 92]

Para entender el significado de (41), nos ayudaremos del par de ejemplos de (42). Nos detendremos en comprender qué designa el SN el ganador de las elecciones en (42b) y si los sintagmas nominales Trump y el ganador de las elecciones son expresiones sinónimas en (42a) y (42b). 
(42) a. Trump visitó el instituto

b. El ganador de las elecciones visitó el instituto

Esta parte del ejercicio sirve para mostrar que los SN denotan entidades y las oraciones, hechos. En (42b) el SN el ganador de las elecciones denota una persona, por lo que puede ser sustituido por un nombre propio, como en (42a). Por su parte, el predicado visitar debe tener como sujeto un SN que denote una entidad. Como las oraciones no denotan individuos, (43) es agramatical:

(43) *Que ganara las elecciones visitó el instituto

Sin embargo, el SN el ganador de las elecciones en otro contexto sintáctico, como el del ejemplo de (41), tiene dos posibles interpretaciones. Para ver ambas lecturas, es necesario contextualizar o parafrasear el ejemplo. Una de las lecturas es nominal (44b) y otra, que no se espera, es oracional (44a). En las paráfrasis se ve que el SN el ganador de las elecciones equivale a una oración ('quién fue el ganador') en (44a) y a un SN ('Trump') en (44b). ${ }^{24}$

(44) a. Me sorprendió quién fue el ganador, no me lo esperaba para nada

b. $\{\mathrm{El}$ ganador/Trump\} me sorprendió con sus tuits racistas

Para descubrir las regularidades y los límites del fenómeno, hay que fijarse en las condiciones de selección entre predicados y argumentos. Ya vimos que algunos verbos exigen oraciones (preguntar) y otros verbos exigen sintagmas nominales de complemento (conocer):

(45) a. Juan me preguntó quién había ganado las elecciones

b. *Juan me preguntó Trump

(46) a. *Juan conoció \{que Trump había ganado las elecciones / quién había ganado...\}

b. Juan conoció a Trump

Sin embargo, en (47) los verbos preguntar y conocer aparecen con un complemento nominal, lo que no es esperable dadas las restricciones de selección de preguntar:

(47) a. Juan me preguntó el ganador de las elecciones

b. Juan conoció al ganador de las elecciones

En principio, no hay nada en el SN el ganador de las elecciones que nos haga pensar que esta secuencia se pueda interpretar como una pregunta. Habría que explicar, por tanto, por qué no existe con las preguntas encubiertas una relación entre forma y significado. Para que los alumnos se percaten de este desajuste, podemos formular una serie de cuestiones que les ayuden a descubrir qué diferencia hay entre (47a) y (47b):

1) ¿Dado que el SN el ganador de las elecciones tiene la misma distribución en (47a) y (47b), deberíamos deducir que este SN significa lo mismo en (47a) y (47b)?

2) ¿A partir del significado de los verbos preguntó/conoció y el SN el ganador de las elecciones se puede construir el significado de la oración? 
3) ¿Qué hay que añadir en (47a) para obtener lo que significa el ejemplo ('Juan me preguntó quién era el ganador de las elecciones')?

4) ¿Por qué en Conoció al ganador de las elecciones no hay que añadir nada para obtener el significado de manera composicional?

De ejemplos como (47a) no podemos deducir que el verbo preguntar seleccione un SN, así que, en este caso, a diferencia de lo que sucede en (47b), solo podemos obtener su significado si asumimos que hay un significado de pregunta que está oculto y que satisface los requisitos de selección del verbo preguntar.

Por último, las preguntas ocultas nos permiten reflexionar sobre la diferencia entre incorrección y agramaticalidad. Examinemos la anomalía del titular de periódico de (48): ¿cómo es posible que el sujeto (las ofertas que rechazó) no concuerde con el verbo (es)?

(48) El mánager general de los Rockets alucina con Llull: "Es increíble las ofertas que rechazó" [19/04/2018, OK Diario]

Como hemos visto a lo largo de nuestro artículo, la discordancia se explica si pensamos que el $\mathrm{SN}$ se interpreta como una oración, ya que, cuando las oraciones son sujeto, el verbo principal aparece en singular: ${ }^{25}$

(49) Es asombroso que haya ganado las elecciones

La reflexión sobre las discordancias ayudará a que los estudiantes entiendan que la gramática no es un sistema impuesto y que ciertos "errores" tienen un trasfondo gramatical. Podemos también hacerles entender que la actitud del gramático no ha de ser prescriptiva y que podemos intentar buscar una explicación a los datos anómalos. Lo interesante ante datos como los de (48) no es decir que son incorrectos porque no hay concordancia, sino más bien preguntarnos por qué es posible que no exista concordancia y la oración resulte gramatical.

\section{CONCLUSIONES}

En este trabajo hemos hecho una propuesta sobre cómo se pueden utilizar las preguntas encubiertas para mejorar la enseñanza de la gramática. Sostenemos que el hecho de que se trate de SN con significado proposicional, algo que no es esperable, puede emplearse en secundaria para enseñar, de manera práctica y no teórica, principios básicos de la gramática como la composicionalidad y la selección (categorial y semántica), o la diferencia entre gramaticalidad y corrección. Nuestra propuesta se enmarca en una línea de investigación sobre la enseñanza de la gramática que promueve el estudio de la lengua partiendo de la intuición que los hablantes tienen sobre ella, de manera que se llegue a la forma desde el significado, con una metodología nueva que promueva la reflexión, el análisis empírico y la realización de generalizaciones.

\section{NOTAS}

1 El término pregunta encubierta engloba también la interpretación exclamativa. El análisis de estos sintagmas tiene una larga tradición en los estudios hispánicos: Andrés Bello y Rufino José Cuervo ya trataron estas construcciones. En la tradición generativista, los primeros trabajos sobre 
preguntas encubiertas se remontan a Baker (1968) y Heim (1979). Estudios más recientes son los de Bosque, 1984; Nathan, 2006; Romero, 2006; Vatrican, 2006; Bosque, 2017b; Frana, 2017; Gutiérrez-Rexach y Andueza, 2017, y Vatrican, 2019, entre otros.

2 En Bosque (1989, 94) se habla también de un tercer significado, modal: Es necesaria una reparación 'Es necesario hacer una reparación'. Sin embargo, a diferencia de las lecturas interrogativa y exclamativa, el significado modal parece estar exclusivamente en el predicado y no en su complemento. Por ello, Es necesaria una reparación no equivale a Es necesario poder hacer una reparación (frente a, por ejemplo, las interrogativas indirectas de infinitivo, de las que también se dice que tienen significado modal: No sé qué hacer 'No sé qué puedo hacer'). Reservamos el estudio de este tipo de sintagmas para otros trabajos.

3 Por eso se dice que los predicados que introducen exclamativas son predicados factivos, que presuponen la verdad de su complemento. El complemento es verdadero, y el hablante introduce una evaluación positiva o negativa sobre él. Sobre esta cuestión, véase Bosque (2017b, 44).

4 Tal y como se recoge en el capítulo introductorio de Frana (2017), para Nathan (2006) y Romero (2006) la interpretación de las interrogativas ocultas se limita a oraciones atributivas de identidad (Sabía el autor 'Sabía que el autor era x'). Estos trabajos se ocupan únicamente de SN simples.

5 Este sintagma tiene también la lectura referencial en la que el sintagma no tiene significado proposicional.

6 Como se señala en Leonetti (1999, 827), para que la lectura cuantitativa se dé el sustantivo debe aceptar la cuantificación. Por ello, en singular un SN con un nombre contable solo tiene la interpretación cualitativa: Me sorprendió el libro que leía 'Me sorprendió qué libro leía'. Con un nombre no contable se recupera en singular la lectura cuantificativa: Me sorprendió el tiempo que tardó 'Cuánto tiempo tardó'. El significado del sustantivo también puede favorecer las lecturas cuantificativas o las cualitativas. Así, contrastan Ya ves el agua que bebe, que tiene lectura cuantitativa 'cuánta agua' y cualitativa 'el agua tan mala que bebe', y Ya ves la vida que lleva, que toma únicamente una interpretación cualitativa.

7 En la lectura cuantitativa, la orientación de la escala parece ser siempre positiva, es decir, Es increíble los libros que lee equivale a 'los muchos libros', igual que cuántos equivale a 'qué muchos'. Para obtener la lectura de 'qué pocos' hay que recurrir a sintagmas del tipo Es increíble los pocos libros que lee 'Qué pocos libros lee'.

8 Como nos indica un revisor anónimo, las preguntas encubiertas complemento de predicados interrogativos pueden tener marginalmente significado cuantificativo, como en No sé los hermanos que tiene 'No sé cuántos hermanos tiene' o en ?Me pregunto los días que faltan para las vacaciones. Nótese que, como es esperable, en este caso la lectura cuantificativa no expresa valoración extrema, que se asocia con los predicados exclamativos. Así, la oración Me sorprende los hermanos que tiene en la lectura cuantitativa equivale a 'Me sorprende los muchos hermanos que tiene', mientras que en No sé los hermanos que tiene, la paráfrasis 'No sé los muchos hermanos que tiene' no es posible.

9 Sin embargo, como se señala en Bosque (2017a, 29), los SN que rechazan preguntas encubiertas pasan a admitirlas cuando contienen ciertos modificadores, como oraciones de relativo (Le preguntó la sopera que le gustaba; Decidieron la cama que comprar) o adjetivos que dan lugar a la lectura superlativa (Juan sabe la mesa mejor para esa habitación).

10 Según Bosque y Gutiérrez-Rexach (2009, 719-720), los nombres que pueden funcionar como interrogativas encubiertas se caracterizan por expresar "atributos prototípicos o representativos de las cosas" (No sé la marca de su chaqueta frente a *No sé el bolsillo de su chaqueta) o "rasgos que es habitual suponer en los individuos: Se desconoce su religión, su profesión, sus planes, sus dolencias". En inglés se usa what en lugar de which con estos sustantivos (What is your name?).

11 En la NGLE, § 42.16g se señala que no se puede hablar propiamente de discordancia en estos casos porque si el complemento es oracional, se espera que la concordancia se haga en singular. 
12 Como nos señala un revisor anónimo, lo mismo ocurre en otros casos de discordancia, como con ciertos sujetos coordinados, que concuerdan más frecuentemente en singular si van pospuestos (NGLE, § $33 \cdot 7 \mathrm{n}, \mathrm{r}$ ).

13 Ejemplo tomado del Corpus del Español de Mark Davies [https://www.corpusdelespanol.org].

14 Como hemos visto, es esencial que aparezca el artículo definido (y no otro determinante definido) para que la lectura oracional se dé. Las mismas propiedades cuantificativas tiene, por ejemplo, el artículo neutro cuando se usa como cuantificador de adjetivos y adverbios (NGLE, § 14.9, 42.16ñ). Torrego (1988) estudia algunas construcciones en las que el artículo determinado tiene valores enfáticos o cuantificativos (iLas cervezas que consume la gente en este país!). En el caso de las preguntas encubiertas, la propuesta de Torrego (1988) es que el artículo determinado absorbe el rasgo [+Qu-] - presente en un operador nulo- que seleccionan los verbos de pregunta.

15 En Lauwers y Willems $(2011,49)$ se señala que el término coacción, usado en sentido amplio, engloba una serie de procedimientos semánticos diversos, como desplazamiento de tipo (ing. typeshifting), acomodación (ing. accommodation) o composición enriquecida (ing. enriched composition). En nuestro trabajo hablamos de coacción cuando el selector impone requisitos al elemento seleccionado para reparar un desajuste que se ha producido en un contexto sintáctico particular. En las construcciones "coaccionadas" se produce un efecto semántico como resultado de la estrategia de reparación. Un ejemplo clásico de coacción del complemento lo encontramos en la oración John began the book (esp. Juan empezó el libro) (Lauwers y Willems, 2011; Jackendoff, 1997; Pustejovsky, 1995). El verbo empezar coacciona al complemento nominal y hace que este SN pase de denotar un objeto ('libro') a interpretarse como un evento ('leer un libro').

16 Para las diferentes propuestas de cómo enriquecer el significado de algunas expresiones, véase Bosque (2000). En este artículo se tratan expresiones sincategoremáticas, es decir, expresiones cuyo significado no se deriva del contenido de su entrada léxica. Un ejemplo es la construcción formada por adverbios que toman como complementos sustantivos que no denotan eventos, pero que se interpretan como si los denotaran: después del último autobús ('después de que pasara el último autobús'). La pregunta que se plantea es si el significado extra está codificado en el léxico o se obtiene mediante procedimientos semánticos y pragmáticos.

17 El efecto interpretativo que se produce es sistemático y predecible (Escandell-Vidal y Leonetti, 2011).

18 El significado de una expresión lingüística es el resultado de lo que significan las partes que la componen y de su sintaxis, es decir, de los procesos por los cuales se combinan esas partes.

19 La selección semántica se asocia normalmente (pero no solo) con el papel temático (agente, paciente, tema, meta, proposicional, etc.) del argumento, mientras que la selección categorial tiene que ver con el tipo de sintagma (nominal, oracional, etc.) que es el argumento.

20 La asimetría que reflejan los ejemplos anteriores tiene, como señala Bosque $(1989,85)$, una base semántica, ya que "las entidades que designan las proposiciones pueden ser objeto de conocimiento, de comunicación y de experiencia. Pueden ser lamentadas, creídas, afirmadas o ignoradas, pero no compradas, fumadas ni conducidas". Existe una correlación entre la selección c(ategorial) y la selección s(emántica), pues podemos derivar la categoría gramatical a partir del significado de una expresión.

21 Como nos indica un revisor anónimo, el verbo saber sí acepta SN de complemento en un empleo: Sabe un poema de Espronceda, sabe la lección. En la NGLE, § 28.4g se señala que este predicado tiene dos significados distintos. Con uno de ellos equivale a 'tener certeza' y se construye con oraciones; con el otro significado equivale a 'poseer capacidad o habilidad', como en Saber cantar. Con este segundo significado, el verbo admite sustantivos que denoten materias de conocimiento.

22 En el Real Decreto 1105/2014 encontramos afirmaciones como la siguiente: "El bloque [Conocimiento de la lengua] responde a la necesidad de reflexión sobre los mecanismos lingüísticos que regulan la comunicación y se aleja de la pretensión de utilizar los conocimientos lingüísticos 
como un fin en sí mismos para devolverles su funcionalidad original: contribuir al desarrollo de la capacidad del razonamiento lógico y servir de base para el uso correcto de la lengua".

23 Para saber más sobre esta asociación, véase su página web: https://sites.google.com/view/groccat/home.

24 Conviene que los alumnos se den cuenta de que este comportamiento de los SN no es un hecho aislado, sino que es una propiedad del sistema y que está sujeto a ciertas restricciones. Para ello, se les puede pedir que creen ejemplos similares a los que estamos analizando, en los que el SN subrayado reciba una interpretación referencial y una lectura de pregunta encubierta, como $\mathrm{Ha}$ olvidado la capital de Italia o Recuerda la calle de su colegio.

25 Otra cuestión interesante que se podría explotar al estudiar este tipo de ejemplos es la de la concordancia. En concreto, se puede hablar del tipo de relación que se establece entre la oración que es sujeto (que haya ganado las elecciones en (39)), y el verbo, pues el sujeto concuerda en número y persona, pero las oraciones no tienen ni número ni persona. Esto se puede vincular con oraciones del mismo tipo del inglés (it was great to have you here), en las que aparece un pronombre expletivo (it), de manera que el sujeto "se divide" entre dos posiciones.

\section{REFERENCIAS BIBLIOGRÁFICAS}

BAKER, C. L. 1968. Indirect questions in English. Tesis doctoral. Urbana, IL: University of Illinois. BOSQUE, I. 1982. "Sobre la interrogación indirecta", Dicenda. Cuadernos de Filología Hispánica, 1 , pp. 13-34.

BOSQUE, I. 1984. "Sobre la sintaxis de las oraciones exclamativas", Hispanic Linguistics, 1, pp. 283-304.

BOSQUE, I. 1989. Las categorías gramaticales. Madrid: Síntesis.

BOSQUE, I. 2000. "Objetos que esconden acciones. Una reflexión sobre la sincategorematicidad”, en Cabré, M. T. y Gelpí, C. (eds.), Lèxic, corpus i diccionaris. Cicle de conferències $i$ seminaris 97-98. Barcelona: Institut Universitari de Lingüística Aplicada, Universitat Pompeu Fabra, pp. $15-30$.

BOSQUE, I. 2017a. “Sobre los verbos de memoria y la interpretación semántica de sus argumentos”, Lingüística, 33(1), pp. 9-32.

BOSQUE, I. 2017b. "Spanish exclamatives in perspective: a survey of properties, classes, and current theoretical issues", en Bosque, I. (ed.), Advances in the analysis of Spanish exclamatives. Columbus, $\mathrm{OH}$ : The Ohio State University Press, pp. 1-52.

BOSQUE, I. y GALLEGO, Á. J. 2016. "La aplicación de la gramática en el aula. Recursos didácticos clásicos y modernos para la enseñanza de la gramática”, Revista de Lingüística Teórica y Aplicada, 54, pp. 63-83.

BOSQUE, I. y GUTIÉRREZ-REXACH, J. 2009. Fundamentos de sintaxis formal. Madrid: Akal.

BOSQUE, I. y MORENO, J. C. 1990. "Las construcciones con lo y la denotación del neutro", Lingüística, 2, pp. 4-50.

BRUCART, J. M. 1999. "La estructura del SN: las oraciones de relativo”, en Bosque, I. y Demonte V. (dirs.), Gramática descriptiva de la lengua española. Madrid: Espasa, pp. 395-522.

BRUCART, J. M. 2014. "Nociones básicas de gramática y su aplicación a la enseñanza”. Sesión en el curso Qüestions de gramàtica actual: perspectives i aplicacions, 4 de julio, Barcelona.

ESCANDELL, M. V. y LEONETTI, M. 2011. "El estudio de la lengua: comunicación y gramática", en Ruiz Bikandi, U. (coord.), Lengua castellana y literatura: complementos de formación disciplinar. Barcelona: Graó, pp. 61-80. 
ESCANDELL-VIDAL, V. y LEONETTI, M. 2011. "On the rigidity of procedural meaning”, en Escandell-Vidal, V., Leonetti M., y Ahern A. (eds.), Procedural meaning. Problems and perspectives. Bingley: Emerald, pp. 81-102.

FRANA, I. 2017. Concealed questions. Oxford: Oxford University Press.

GRIMSHAW, J. 1979. "Complement selection and the lexicon". Linguistic Inquiry, 10(2), pp. 279-326.

GUTIÉRREZ-REXACH, J. y ANDUEZA, P. 2017. "Embedded exclamatives and the ingredients of grounded belief", en Bosque, I. (ed.), Advances in the analysis of Spanish exclamatives. Columbus, OH: The Ohio State University Press, pp. 181-210.

HARRIS, J., PYLKKÄNEN, L., MCELREE, B., y FRISSON, S. 2008. "The cost of question concealment: eye-tracking and MEG evidence”, Brain and Language, 107, pp. 44-61.

HEIM, I. 1979. "Concealed questions", en Bauerle, R., Egli U., y Stechow, A. (eds.), Semantics from different points of view. Berlín: Springer, pp. 51-60.

JACKENDOFF, R. 1997. The architecture of the language faculty. Cambridge, MA: MIT Press.

LAUWERS, P. y WILLEMS, D. 2011. "Coercion: definition and challenges, current approaches, and new trends”, Linguistics, 49, pp. 1219-1235.

LEONETTI, M. 1999. "El artículo", en Bosque, I. y Demonte V. (dirs.), Gramática descriptiva de la lengua española. Madrid: Espasa, pp. 787-890.

LEONETTI, M. 2017. "La importancia de la relación entre forma y contenido". Comunicación presentada en Jornadas GrOC Madrid, 19-20 de octubre, Madrid.

NATHAN, L. 2006. On the interpretation of concealed questions. Tesis doctoral. Cambridge, MA: MIT.

PLANN, S. 1982. "Indirect questions in Spanish", Linguistic Inquiry, 13(2), pp. 297-312.

PUSTEJOVSKY, J. 1995. The generative lexicon. Cambridge, MA: MIT Press.

RAE-ASALE. 2009. Nueva gramática de la lengua española. [NGLE]. Madrid: Espasa.

ROMERO, M. 2006. “On concealed questions”, en Gibson M. y Howell, J. (eds.), Proceedings of semantics and linguistic theory (SALT) XVI, Ithaca, NY: Cornell University, pp. 208-227.

TORREGO, E. 1988. "Operadores en las exclamativas con artículo determinado de valor cuantitativo”, Nueva Revista de Filología Hispánica, 36, pp. 109-122.

VATRICAN, A. 2006. "Un caso de 'desaparición' lingüística: el verbo saber y las preguntas encubiertas", Revue Babel, 13, pp. 265-286.

VATRICAN, A. 2019. "Acerca de las preguntas encubiertas 'concealed questions': el dudoso caso de saber en español”. Comunicación presentada en el XLVIII Simposio de la Sociedad Española de Lingüística, 29 de enero - 1 de febrero, Madrid.

\section{* AGRADECIMIENTOS}

Una parte de este artículo se presentó en el XXXVI Congreso de AESLA, Cádiz 2018. Agradecemos al público asistente sus comentarios y sugerencias, que han contribuido a mejorarlo. Este trabajo ha sido financiado parcialmente por el proyecto SPIRIM (Ref. FFI2015-63497-P).

\section{NOTA SOBRE LAS AUTORAS}

Edita Gutiérrez Rodríguez es doctora en Lingüística y profesora en la Universidad Complutense de Madrid, España.

Pilar Pérez Ocón es doctora en Lingüística y profesora en la Universidad de Castilla-La Mancha, España. 